\title{
Study on the construction of domain-specific ontology in oil field
}

\author{
Xiaoya Deng \\ School of Computer, Sichuan University of Arts and Science, Dazhou 635000, China
}

dxy14@163.com

Keywords: oil field, ontology representation, domain-specific ontology, micro-ontology, Ontology construction.

\begin{abstract}
This article firstly introduces the theory of construction of domain-specific ontology and oil ontology business framework model, and then puts forward the method to construct the oil exploration and development of ontology library, the exploration and development of oil field involved is divided into multi-level business activity levels, and takes reservoir-seal combination activity as an example and lists to explore the construction method of the corresponding micro-ontology library, at last constructs a complete petroleum domain-specific ontology model.
\end{abstract}

\section{Introduction}

With the development of petroleum exploration and technology, the wide spread of Internet, various amount of related information greatly enriched, cross disciplinary knowledge growth, more and more problems appear in the field of oil exploration. The field of oil exploration and development including exploration, drilling, oil test, production engineering, petroleum storage and transportation and so on more than twenty professional, which cooperate and compose the entire process of the petroleum exploration. But it is a common phenomenon that the simple pursuit of realization of function, not from the whole industry level to plan the design and development of the development and application of various kinds of petroleum exploration and the use of the system, making the beginning to many aspects of organization and management of data by inconsistent with the understanding and use of various professional knowledge requirements identified from the research. Causes between the various systems of information and knowledge sharing obstacles, cannot provide a perfect global field of oil exploration and development solutions. So in the long run the global field of oil exploration and exploitation of knowledge building has become a trend, unified comprehensive management and decision-making of the exploration and exploitation of petroleum resources from the point of view of knowledge, the establishment of ontology base oil field for the oil company build into a competitive advantage essential link.

\section{Construction of Ontology}

Ontology. At the beginning of the twentieth century, some scholars use "ontology" this Chinese word translation of western philosophy in pursuit of "beyond all existence or phenomenon, with the ability to create all kinds of things or phenomena". With the development of computer science, the computer science community to use the term "ontology" describes proposed by the Gruber: An ontology is the standardization of the conceptualization of the clear instructions [1-4].

Research and application of ontology relates to the intelligent information integration, knowledge engineering, Natural Language Processing, software engineering and so on. And how to extract these different domain knowledge and represent of domain-specific ontology and construct appropriate has become the hot spot of the current research [5-7].

The so-called domain ontology is a professional ontology, including the concept, the concept of subject, the relationship between the attributes of concepts and attributes and relationships between constraints. Because knowledge has the significant characteristics of the field, so the domain-specific ontology can be represented more effective knowledge. Domain ontology is usually used to represent 
special knowledge in a particular field within the scope of construction method for example by this text is the study of petroleum exploration and development of the domain ontology as the main content.

Construction of Ontology. The structure of the ontology is one of the five tuple $O:=\{C, R, H c$, Rel, Ao\}. A semantic basis as between different subjects to communicate, the body is composed of a specific term describing a certain reality sets, plus a set of explicit terminology connotations. In the simplest case, a classification hierarchy ontology only describe the concept: in the case of complex, can be based on ontology concept classification levels, adding a suitable set of relations, axioms, rules to represent other relationships between concepts, explains the connotation of the concept of constraint. A complete ontology should be made of five kinds of basic elements as concept, relation, function, axioms and examples. Ontology includes a series of classes or concepts which are the core of the ontology. The definition of general use frame structure, including concept name, the relationship between concept, and the use of natural language description of the concept.

\section{Oil ontology business framework model}

Business Framework Model. The key step of building the domain ontology model firstly lies in the establishment of domain ontology framework suitable model. The oil ontology business model is a kind of defined composition by logical relationship between activities and activities to describe the production process of petroleum enterprise management model.

Division of Business Domain. Division of business domain in some oil related topics as guidance for the overall oil main business division. It is not a copy of existing agencies, nor the basic business consolidation, but the summary, summary of the division [8-10].

The division of business domain can be divided in accordance with the business management form or oil life cycle of organization. For example, according to the business management can be divided into "exploration, development, production, management" of four categories.

Business domain delineation method of life cycle as the main line, professional business domain and service management method organic serialized accords with the management of oil and gas exploration and development of the conventional management habits, do different business domains between business does not repeat, and guaranteed to cover all of the business of exploration and development.

Based on the above principles and methods, the oil and gas exploration and development business is divided into eight business domains. With "comprehensive research" business division as an example, as shown in Table 1, first of all, the comprehensive research in the exploration and development of life cycle in the later stage of exploration, but also a key stage, the professional characteristics of it is so outstanding that the generated document chunks of data and a lot of results, so the "comprehensive research" to treat as a top business domain. Below it contains five primary business: resource evaluation, structure research, study of remaining oil and reservoir simulation, reservoir description and evaluation; And in one of the "Resource evaluation " as an example, it contains four level two business: basin evaluation, zone evaluation, exploration evaluation and prediction of reserves calculation ; And in one of the "zone evaluation", it contains five three level of business activity: evaluation of hydrocarbon source rock condition, evaluation of accumulation condition, evaluation of reservoir condition, evaluation of preservation condition and evaluation of trap condition. 
Table 1 Division of comprehensive research business

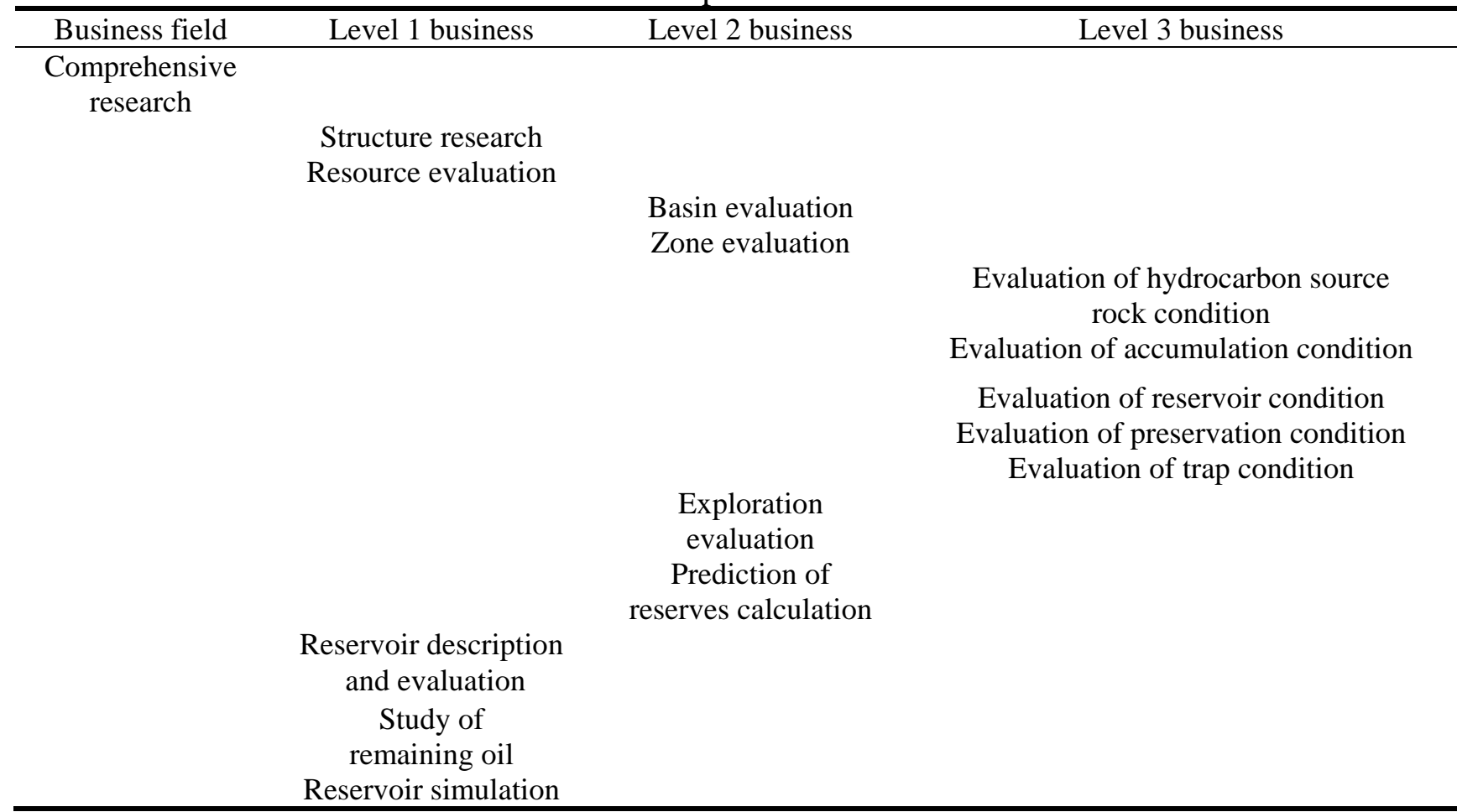

Ontology Model of Business Activities. Business activity is the most basic unit of the minimum function, cannot be broken business process decomposition. Generally, a business process comprises a plurality of business activities. Division and business analysis from the perspective of the business activities is relevant.

Business activity description: summary text of business activity description, description is described in accordance with the "6W" model, namely the activity is by who initiated, at what time initiated, where initiated, why to initiate this activity, in this activity are related to what objects, what are the characteristics of objects. Through the "6W" process, can be a business related information relates to completely extract.

\section{Taking the Reservoir-seal combination of ontology construction as an example}

An ontology model in the field of one must first determine the business theme, in the relevant business topics under the guidance of the collection and collation of the business within the scope of all the vocabulary and terminology, and then according to the lexical level rationally divide the word set, with a relationship between these lexical organization structure model of the tree, the word set based to improve the finishing between the upper concept, to establish these attributes of vocabulary, operation process and criteria for identification and lexical relations, evaluation techniques and parameters, has formed a complete domain ontology model.

The Reservoir-seal combination of micro-domain ontology model is shown in Figure 1.

\section{Conclusions}

In this paper, through theoretical discussion and Research on the construction of domain ontology, proposes a construction method of the field of petroleum exploration and development of ontology, and with the reservoir-seal combination activity lists corresponding micro ontology model as an example. It can be predicted: in the near future, the construction and application of the progress of human society will made great contribution to the field of ontology. 


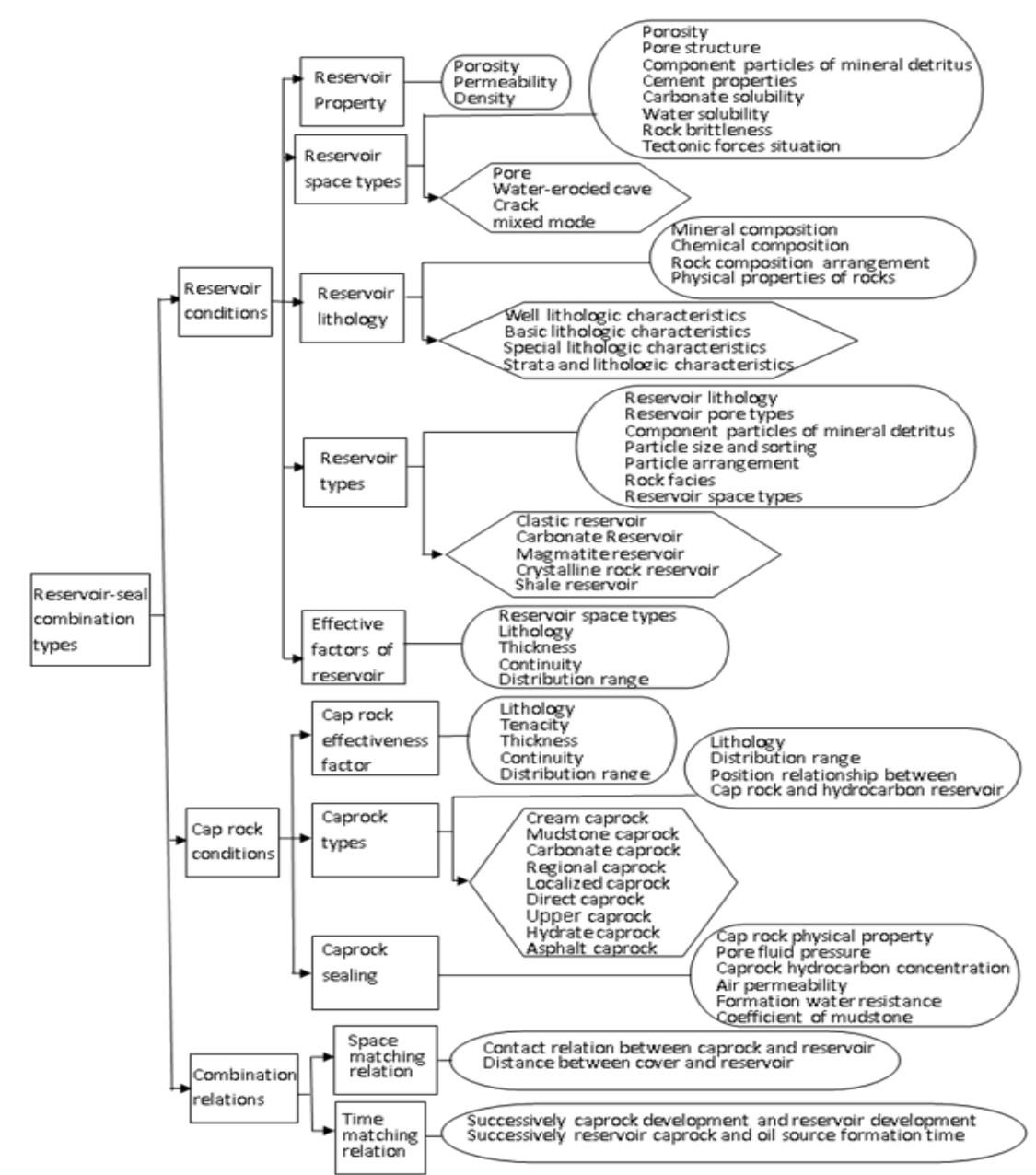

Fig. 1 Reservoir-seal combination of micro-domain ontology model

\section{References}

[1] Thomas R Grube: Knowledge Acquisition, (1993) No. 2, p.199-200.

[2] Bomt W N. Construction of Engineering Ontologies for Knowledge Sharing and Reuse (Ph.D., University of Twenty, Holland 1997).

[3] Guoming Yuan, Hongqi Li and Bo Fan: The 2011 IEEE International Conference on Electronics and Optoelectronics (Dalian, China, April, 2011).p.401-403.

[4] Gang Chen, Ruqian Lu, Zhi Jin: Journal of Software, (2003) No.14, p.350-355. (In Chinese)

[5] Guoming Yuan, Hongqi Li: Microcomputer \& Its Applications, (2010) No.29, p. 5-7.(In Chinese)

[6] Fridman Noy and CD Hafher: AI Magazine, (1997), p.53-74.

[7] Guarino N: Proceedings of the lst International Conference on Formal Ontology in Information Systems (Trento, Italy, 1998), p.3-15.

[8] Peter Mika: Web Semantics: Science, Services and Agents on the World Wide Web, (2007) No.1, p. 5-15.

[9] Baohe Liu: Encyclopedia of China Petroleum Exploration and Development (Petroleum industry press, China 2008).

[10] Guoming Yuan, Hongqi Li and Bo Fan: Computing technology and automation, Vol. 30 (2011) No.1, p.138-143.(In Chinese) 